\title{
3D Model Identification Using the Dice-Shape Flat Pattern
}

\author{
Jeongseok Jo, Yongbae Kim, and Jongweon Kim
}

\begin{abstract}
In this paper, we introduce 2D view-based method by using the combined depth image of the dice-shape flat pattern, scale-invariant feature transform (SIFT) algorithm and random sample consensus (RANSAC) algorithm. The combined depth images are generated by the direction of a square face views and these features are composed in the feature DB. We previously did not use the combined depth image of the dice-shape flat pattern but that of the tile-shape. We compare the combined depth image of the dice-shape flat pattern with that of the tile-shape. The combined depth images of the dice-shape flat pattern are composed in the 6 depth images and also that of the tile-shape. In the experiment, we use the number of 16 classes in the SHREC benchmark database and there are 3 to 4 the similar 3D models in the class.
\end{abstract}

Index Terms-Dice-shape flat pattern, SIFT, RANSAC, tile-shape, depth image, 3D model identification.

\section{INTRODUCTION}

As a patent for the 3D printer gradually expire, the price of 3D printers have been steadily lowered, which may give to accelerate the spread of 3D printer. For this reason, the development of a 3D printer industry is to make possible an individual custom-made for products and a design drawings and a floor plan for the products have been shared on the Internet to enable custom-made. The people making design drawings and a floor plan have also increased by using the computer-aided design (CAD) and 3D MAX. The produced design drawings and a floor plan could allow the replication work produced work based on the thoughts and feelings of the creator though a $3 \mathrm{D}$ printer.

The 3D model retrieval algorithms were about the retrieval of the similar 3D models and they were focused on more accurately retrieval among many similar 3D models. However, it is also considered important that research to find exactly what you are looking for 3D models and even among 3D models of similar shape.

To identify the 3D model among the similar 3D models, we use $2 \mathrm{D}$ view-based method and extract features of the 3D model using SIFT algorithm [1]. To compare more accurately the features in the feature DB with the features of the query model, we use RANSAC algorithm to increase the match rate [2], [3].

If we use the same model (i.e. the query model and the 3D model in the feature DB), the experiment result for 3D model

Manuscript received September 2, 2015; revised November 27, 2015. This research was supported by Institute for Information and Communications Technology Promotion (IITP) funded by the Ministry of Science, ICT and Future Planning (No.R0126-15-1024).

Jeongseok Jo and Jongweon Kim are with the Copyright Protection Department, Sangmyung University, 20, Hongjimun-2gil, Jongno-gu, Seoul, 03016, Korea (e-mail: jeongs329@naver.com,jwkim@smu.ac.kr, ).

Yongbae Kim is with the Creative Content Labs, Sangmyung University, 20, Hongjimun-2gil, Jongno-gu, Seoul, 03016, Korea (e-mail: ybkimdb@gmail.com). identification is $100 \%$ when we compose features in the feature DB using the combined depth image of the dice-shape flat pattern or that of the tile-shape for each of 3D models However, the number of the features for the identified 3D model is not equal.

For example, if the feature DB includes the features of the dog model which have the number of 200 features, we compare these features with the features of the query model (i.e. the dog model). As a result, we can find the dog 3D model because the number of matched features (i.e. 170) is higher than other models. Although the examples find the dog model, it is possible that both the features in the feature DB and that of the query model cannot match all.

In the experiment, we show that the combined depth image of the dice-shape flat pattern is better than that of the tile-shape when the feature DB is composed. The dice-shape flat pattern can be generated by the number of 11 flat patterns. The experimental result is slightly different for each of 11 flat patterns. The Fig. 1 shows the total process about the 3D model identification of the proposed work.

In this paper is divided into 3 sections. The Section I explains the proposed work. The Section II shows the experiment and result. The Section III is the conclusion.

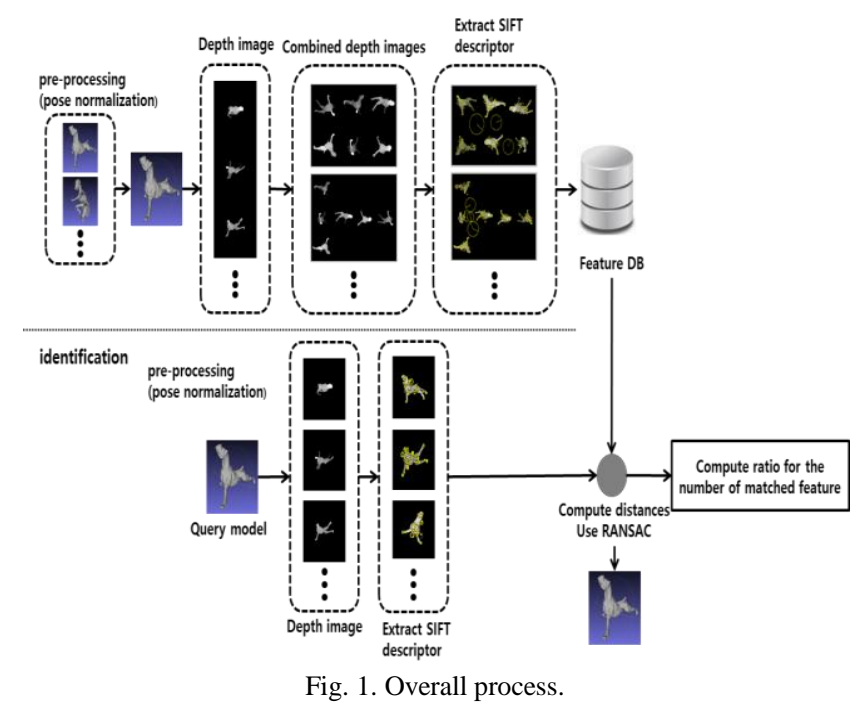

\section{PROCEDURE FOR PAPER SUBMISSION}

\section{A. Overall Process}

The proposed method gets 6 depth images for 3D model and each of depth images is 256 by 256 size as Fig. 2 [4]. We get the depth images from the 3D model as close as possible to increase the resolution of depth images. The overall process is as follows;

\section{1) Pose normalization}

Pose Normalization makes 3D model to scale, rotation, translation invariant form using Principal Component 
Analysis (PCA) and distance normalization. The distance normalization makes the distance to 1 length from the center of gravity for 3D model to the farthest vertex [5], [6].

\section{2) Generate combined depth image}

We used the combined depth image of the tile-shape in the previous method [7]. In this paper, we also use the dice-shape flat pattern form and compare the former method with the latter method.

\section{3) Extract SIFT features}

The SIFT features have 128-dimentsion and the average number of features for the combined depth image of the tile-shape and the dice-shape flat pattern is different.

\section{4) Feature matching}

After extracting the features for 6 depth images for query model, the features of each of depth images are matched with the features of the combined depth images in the feature DB.

\section{5) Remove outlier by using RANSAC and ranking}

The matching process may match dissimilar features (i.e. outlier). The outlier cause to reduce the match rate. Thus, we use RANSAC algorithm to remove outlier. After RANSAC, we compute the number of the matched features and make the rank. After ranking, we choose the best of the best model in the rank.

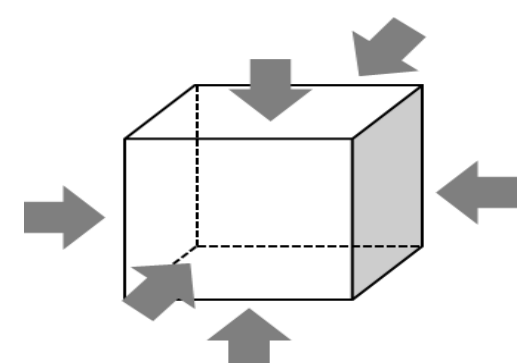

Fig. 1. Generate 6 depth images to 6 viewpoints.

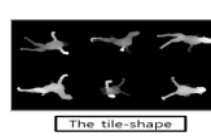

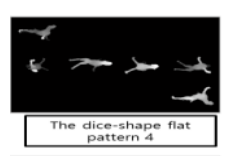
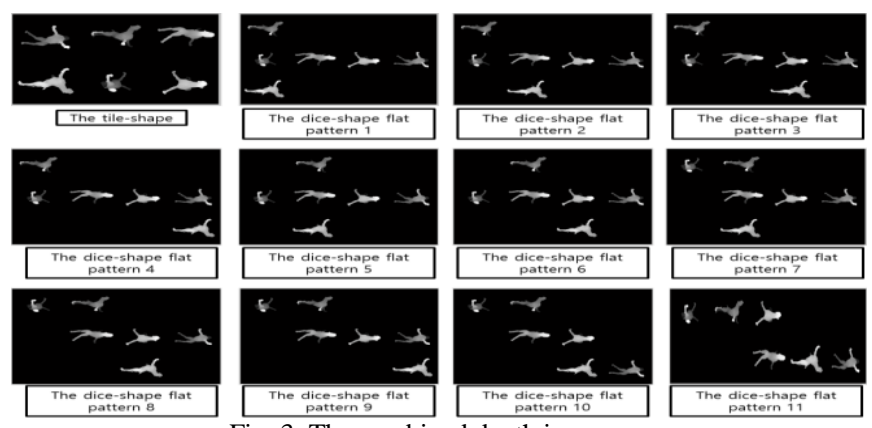

Fig. 3. The combined depth images.

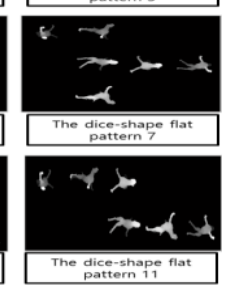

The dice-shape flat
pattern 11

\section{B. Compare the Combined Depth Image of the}

Dice-Shape Flat Pattern with That of the Tile-Shape

The combined depth images (i.e. the dice-shape flat pattern and the tile-shape from) are also generated such as the number of 12 images as Fig. 3. There are several features in Fig. 4. As shown in Fig. 4, there are more features between the depth images. These features will improve the matching rate of the 3D models.

The comparison process is as follows.

1) The number of $\mathrm{N}$ of $3 \mathrm{D}$ models are converted through pose normalization to the normalized 3D models.

2) The normalized models are composed by the combined depth images (i.e. one tile-shape and eleven dice-shape flat pattern). The features $\left(V_{i}, i=1 \cdots N\right)$ in the feature DB are extracted from the combined depth images.

3) The same models used in the feature DB configuration rotate at random to use query model.

4) Since then the features $\left(Q_{i}, i=1 \cdots N\right)$ are extracted to the query model, the features of the query model compare the features $\left(V_{i}, i=1 \cdots N\right)$ in the feature DB.

5) We compute the number of features $\left(V_{i}, i=1 \cdots N\right)$ for each of 3D models in the feature DB and the number of matched features $\left(\right.$ match $\left._{i}, i=1 \cdots N\right)$ after using RANSAC algorithm.

6) The equation is the ratio between the number of features $\left(V_{i}, i=1 \cdots N\right)$ and the number of matched features $\left(\right.$ match $\left._{i}, i=1 \cdots N\right)$ as follows

$$
R_{i}=\frac{\operatorname{match}_{i}}{V_{i}}(i=1 \cdots N)
$$

\section{EXPERIMENT AND RESULT}

In the experiment, we use 3D model of 16 classes in SHREC benchmark database as shown in Fig. 5. There are 3 or 4 of 3D models in a class.

The experimental result shows the average result as the first column of Table I that the number of features differs from each of the combined depth images (i.e. one tile-shape and eleven dice-shape flat pattern).

The features of matched model show the average result as the second column of Table I. We compare the number of features (i.e. extracted from the combined depth images for matched models) with the number of matched features for query model to show the average result as the third column of Table I.
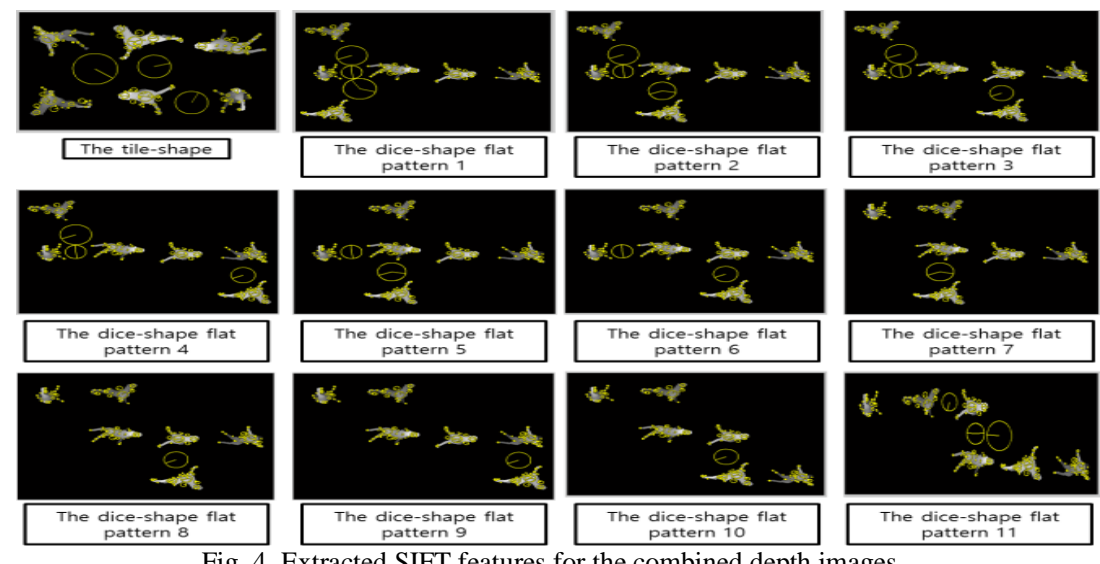

Fig. 4. Extracted SIFT features for the combined depth images. 
The Table I shows that the method using the combined depth image of the dice-shape flat pattern is better than the method using that of the tile-shape. Especially, the combined depth image of the dice-shape flat pattern of 1 type as Fig. 3 is the best of the best for the result.

The reason (i.e. the method using the combined depth image of the dice-shape flat pattern is better than the method using that of the tile-shape) is that the features using the dice-shape flat pattern match the features of query more than the features of the other. Although the former method extracts less the number of features than the latter method, the former method can match more a number of features than the latter method.

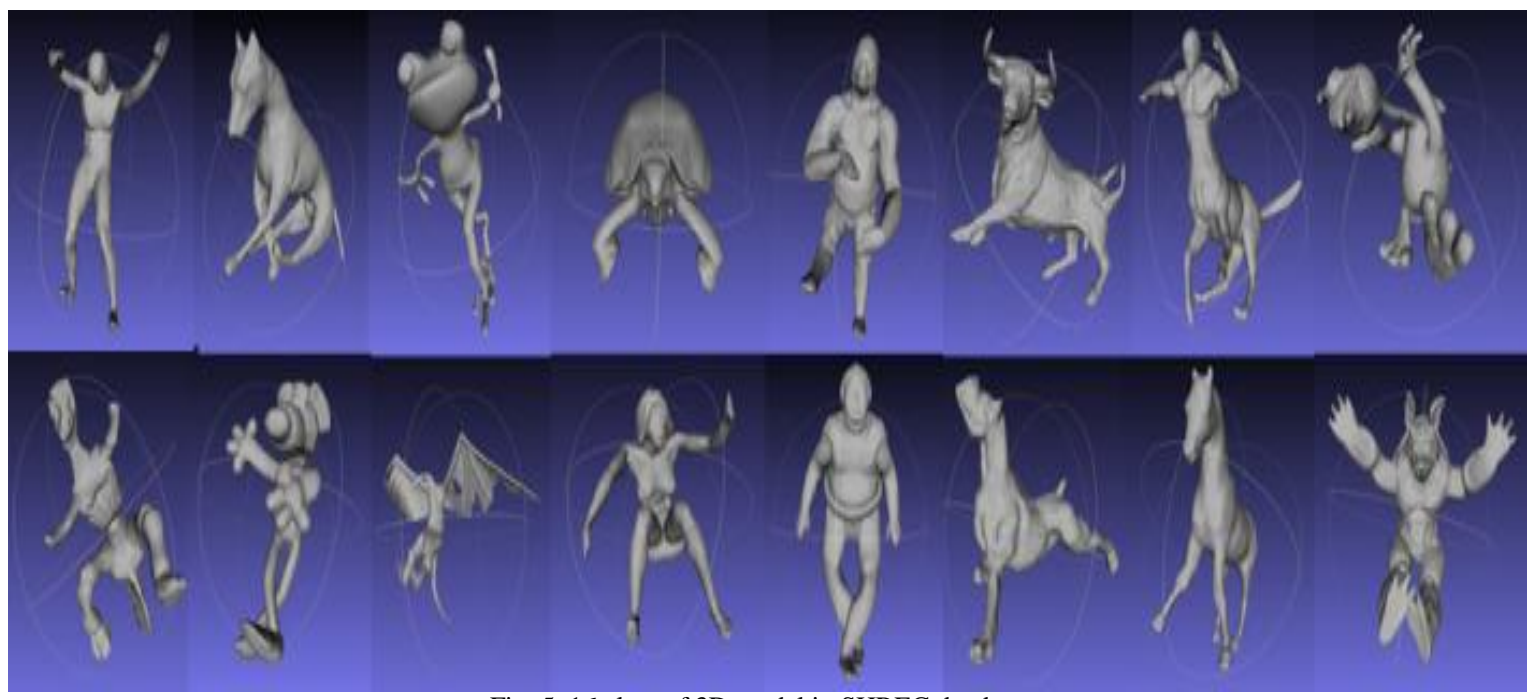

Fig. 5. 16 class of 3D model in SHREC database.

TABLE I: EXTRACTED THE NUMBER OF FEATURES (FEATURE DB)

\begin{tabular}{|c|c|c|c|c|}
\hline \multirow{3}{*}{$\begin{array}{l}\text { Process } \\
\text { number }\end{array}$} & \multicolumn{4}{|c|}{ The shape of the combined depth images } \\
\hline & \multirow{2}{*}{ Tile-shape } & \multicolumn{3}{|c|}{ Dice-shape flat pattern } \\
\hline & & 1 & 2 & 3 \\
\hline \#extracted & 216.55 & 213.03 & 213.56 & 213.53 \\
\hline \#matched & 200.79 & 201.73 & 201.69 & 201.71 \\
\hline \#matched rate & 92.72 & 94.69 & 94.41 & 94.46 \\
\hline \multirow{3}{*}{$\begin{array}{l}\text { Process } \\
\text { number }\end{array}$} & \multicolumn{4}{|c|}{ The shape of the combined depth images } \\
\hline & \multicolumn{4}{|c|}{ Dice-shape flat pattern } \\
\hline & 4 & 5 & 6 & 7 \\
\hline \#extracted & 213.03 & 213.87 & 213.84 & 213.64 \\
\hline \#matched & 201.74 & 201.61 & 201.62 & 201.51 \\
\hline \#matched rate & 94.70 & 94.26 & 94.28 & 94.32 \\
\hline \multirow{3}{*}{$\begin{array}{l}\text { Process } \\
\text { number }\end{array}$} & \multicolumn{4}{|c|}{ The shape of the combined depth images } \\
\hline & \multicolumn{4}{|c|}{ Dice-shape flat pattern } \\
\hline & 8 & 9 & 10 & 11 \\
\hline \#extracted & 213.98 & 213.51 & 213.84 & 213.88 \\
\hline \#matched & 201.56 & 201.46 & 201.25 & 200.80 \\
\hline \#matched rate & 94.19 & 94.35 & 94.11 & 93.88 \\
\hline
\end{tabular}

\section{CONCLUSIONS}

In this paper, we proposed a 3D model identification algorithm using dice-shape flat pattern. This pattern yields more features between the depth images and they improved the identification rate. In the experiment, we use 3D model of 16 classes in SHREC benchmark database and the results show the proposed algorithm which uses dice-shape flat image has better performance than the separated 6 images and tile-shape image. The algorithm can extract 2 or 3 more features than tile-shape image and separated images. In the case of part of the 3D model, the proposed algorithm shows superior identification result than other methods.

In the future, the research team is going to improve the speed of the feature extraction and matching for the purposed of the practical use.

\section{REFERENCES}

[1] D. G. Lown, "Distinctive image features form scale-invariant keypoint," Int'l Journal of Computer Vision, vol. 60, no. 2, November 2014.

[2] A. M. Fischler and R. C. Bolles, "Random sample consensus: A paradigm for model fitting with application to image analysis and automated cartography," Comm. of the ACM, vol. 24, pp. 381-395, 1981.

[3] K. G. Derpanis, "Overview of the RANSAC algorithm," York University, Toronto, Canada, 2010.

[4] D. Y. Chen, X. P. Tian, Y. T. Shen, and M. Ouhyoung, "On visual similarity based 3D model retrieval," Computer Graphics Forum, vol 22, no. 3, Blackwell Publishing, Inc, 2003.

[5] K. Sfikas, T. Theoharis, and I. Pratikakis, "ROSy+: 3D object pose normalization based on PCA and reflective object symmetry with application in 3D object Retrieval," International Journal of Computer Vision, vol. 91, no. 3, pp. 262-279, 2011.

[6] D. D. Varnic and D. Saupe, "3D shape descriptor based on 3D fourier transform," presented at the 3rd EURASIP Conference on Digital 
Signal Processing for Multimedia Communications and Services, Scientific Assoc. of Infocommunications, pp. 1-274. 2001.

[7] J. S. Jo and J. Kim "Defomed 3D model identification using combined depth image," in Proc. ICTC 2015, pp. 725-728, 2015.

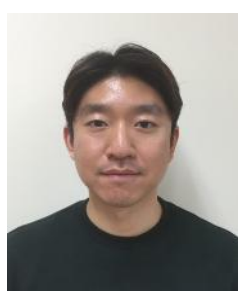

Jeongseok Jo received his B.S degree in intellectual property and computer science from Sangmyung University, South Korea, in 2015. He is currently pursuing the M.S. degree in Copyright Protection, Sangmyung University, South Korea. His research interests are digital watermarking, 3D model identification, Digital forensic.

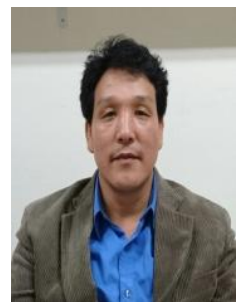

Yongbae Kim was born at Jungsun City, Kangwon, Korea in 1965 and has received the master degree in the signal processing at Chungnam National University, Daejon, Korea in 1989. He has also finished his Ph.D. course at Chungnam National University, Daejon, Korea in 1995. He worked as an assistant professor at Juseong College, Chungbuk, Korea from 1995 to 2010 and He is now a principal researcher of Creative Content Labs at Sangmyung
University, Seoul, Korea. His research interests are signal processing and copyright protection.

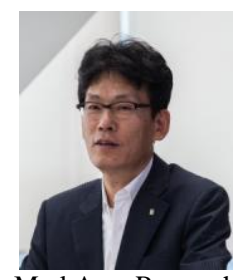

Jongweon Kim was born at Pocheon City, Gyunggi, Korea in 1962 and has received the master and Ph.D degrees in the signal processing at University of Seoul, Seoul, Korea in 1991 and 1995 respectively. He worked as a senior researcher at Korea Institute of Science and Technology Information, Daejon, Korea in 1995, an assistant professor at Juseong College, Chungbuk, Korea from 1996 to 2000, and the head of MarkAny Research Institute, Seoul, Korea from 2000 to 2004, and a research professor and a principal researcher, Copyright Protection Research Institute at Sangmyung University, Seoul, Korea from 2004 to 2009. He is now an associate professor of the Department of Contents and Copyright at Sangmyung University, Seoul, Korea. His research interests are copyright protection, digital watermarking, digital rights management and signal processing. Prof. Kim is a vice president of International Association of Digital Document and The Society of Convergence Knowledge and a member of IEEE, KICS and KSII. 